\title{
Report on the first ASTM Round Robin of Vacuum Panels
}

\author{
ANDRZEJ BRZEZINSKI \\ Lasercomp \\ 20 Spring Street \\ Sangus, MA 01906
}

\section{ABSTRACT}

A round robin was initiated in February of 2000 to compare different methods of determining the effective thermal resistance of vacuum panels. The outcome of this round robin will provide support for the ASTM material specification and the development of a future ASTM test method. Four issues were identified and addressed: (1) calorimetric vs center-of-panel/barrier conductivity approaches, (2) comparison of available finite difference/element models, (3) appropriate boundary conditions for all measurements/models, and (4) comparison of center-of-panel measurements. Six conventional vacuum panels were constructed. All six shared the same dimensional configuration, the same core material, the same getter insert, and the same manufacturing techniques and equipment. Two different barrier materials (three panels from each) were used because barrier thermal conductivity is recognized as a key factor in the determination of effective thermal resistance for vacuum panels, and because the different methods used in this round robin comparison should be sensitive to the barrier thermal properties. The getters were included in these panels to help them remain stable throughout the duration of the round-robin.

Each of the eight participating laboratories measured the center-of-panel resistance of each of the six panels as described in the ASTM standard C1484-00 and reported those results along with pertinent information about the transducer(s) size and location. Several laboratories also determined the whole-panel effective thermal resistance, using two assumed sets of boundary conditions. Considering the exploratory nature of this round-robin, and the complexity of the measurements, the results showed surprisingly good agreement.

\author{
THERESE STOVALL \\ Oak Ridge National Laboratory \\ 1 Bear Creek Road \\ Oak Ridge, TN 37831
}

\section{INTRODUCTION}

Vacuum insulation systems have long been used for cryogenic applications. These systems have historically consisted of multi-layer evacuated jackets with active vacuum systems. In the early 1990s, sealed evacuated panels became commercially available. These panels were filled with either fiberglass or silica and had either metal or plastic barriers. The continuing design evolution includes open-celled foam and advanced powdered fillers, specialty multi-layer films, and the inclusion of new adsorbent systems. In order to help potential users understand the performance of these panels, a task group was formed in 1995 to create an ASTM material specification [1]. Due to the complexity of this non-homogenous insulation form, several evaluation methods were developed by researchers and panel manufacturers. The task group initiated efforts to systematically compare the results of these differing approaches.

The resulting round robin was initiated in February of 2000, with the goal of comparing different methods of determining the effective thermal resistance of vacuum panels. The outcome of this round robin will provide support for the ASTM material specification and the development of a future ASTM test method. Four issues were identified and addressed: (1) calorimetric vs centerof-panel/barrier conductivity approaches, (2) comparison of available finite difference/element models, (3) appropriate boundary conditions for all measurements/models, and (4) comparison of center-of-panel measurements.

\section{ROUND ROBIN DESIGN}

Six conventional vacuum panels were constructed by Dow in January, 2000. All six shared the same dimensional configuration, the same core material, the same getter insert, 
and the same manufacturing techniques and equipment. The specimens were each 12" x 12" by 1", and each was clearly and uniquely marked and evacuated to the same pressure. Two different barrier materials (three panels from each) were used because barrier thermal conductivity is recognized as a key factor in the determination of effective thermal resistance for vacuum panels, and because the different methods used in this round robin comparison should be sensitive to the barrier thermal properties. Aggressive getters were included in these panels to help them remain stable throughout the duration of the roundrobin.

The seven participating laboratories were Advantek, Dow, Dupont, Holometrix, LaserComp, Oak Ridge National Laboratory, and the Product Design Center. Each laboratory measured the center-of-panel resistance of each of the six panels as described in ASTM C1484-00 and reported those results. Several of the laboratories made multiple measurements using different types of apparatus. Pertinent information about the test equipment used to date in this round robin is shown in Table 1. Several laboratories also determined the whole-panel effective thermal resistance, using two assumed sets of boundary conditions.

\begin{tabular}{|c|c|}
\hline Plate size & Central transducer size \\
\hline $24 "$ x 24" & 3" x 3" \\
\hline $24 "$ x 24" & $4 " \times 4 "$ \\
\hline $24 "$ x 24" & $4 " x$ 8" \\
\hline $24 "$ x 24" & $10 "$ x 10" \\
\hline $12 "$ x 12" & $4 " \times 4 "$ \\
\hline $12 " \times 12 "$ & 3" x 3" \\
\hline $12 "$ x 12" & 3" diameter \\
\hline
\end{tabular}

An examination of Table 1 shows that some of the test devices were the same size as the vacuum panels, 12" x 12". In these devices, the entire surface of the vacuum panel was in direct contact with the controlled temperature plate. This configuration therefore represents a constant temperature boundary condition, where the temperature gradient from the center of the panel to the edge of the panel is minimized and the edge heat transfer through the boundary material is reduced. Other test devices were twice as wide as the vacuum insulation panels. For these tests, a high-density fiberglass blanket was sculpted to fit tightly around the vacuum panels and to match the area of the test apparatus plate size. For some of these large plate tests, the vacuum panel was still in direct contact with the constant temperature plate. For others, an arrangement where the fiberglass batt also covers the bottom and top of the vacuum panel was used. When the fiberglass blanket is thus inserted between the constant temperature plates and the vacuum panel, thermocouples are attached directly to the center of the vacuum panel to record the temperature at that location. This last arrangement is typically used with an array of heat flux transducers and is directed more toward measurement of whole panel performance, because it allows a temperature gradient to develop along the face of the barrier material. Despite this limitation, center-of-panel resistivity measurements were also made using this arrangement.

\section{RESULTS}

The measured thermal resistivity values are summarized in Fig. 1. For the panels with a more conductive barrier, the resistivities are all between 26.4 and $31.4 \mathrm{~h} \bullet \mathrm{ft}^{2} \bullet{ }^{\circ} \mathrm{F} / \mathrm{Btu} \bullet$ in. or between 92.3 to $109.7 \%$ of the average value of 28.5 $\mathrm{h} \bullet \mathrm{ft}^{2} \bullet \mathrm{F} / \mathrm{Btu} \cdot \mathrm{in}$. The standard deviation for these ten measurements is $5.1 \%$. For the panels with the less conductive barrier, the resistivities are all between 27.6 and 32.6 , for a range of 92.9 to $109.8 \%$ of the average value of $29.5 \mathrm{~h} \bullet \mathrm{ft}^{2} \bullet{ }^{\circ} \mathrm{F} / \mathrm{Btu} \bullet \mathrm{in}$. The standard deviation for the ten measurements on the less conductive barrier panels is $5.5 \%$.

Previous modeling work on vacuum panels has shown that the center-of-panel measurement will be more accurate for smaller transducer sizes [1]. This is most important if the barrier is more conductive, and the results for this round robin show that effect, as seen in Fig. 2. In this figure, the effect of heat transfer through the panel edges becomes more important as the transducer size approaches the panel size. Indeed, the value measured by a $10 \times 10$ in. transducer is almost the same as the whole panel effective thermal resistance calculated with a finite difference model of the panel (that model used typical heat flux meter apparatus boundary conditions).

The center-of-panel thermal resistivity measurements are also highly dependent on the measured thickness of the panel. For the test configurations, the thickness is automatically measured by the test apparatus. For other test configurations, especially those that employ a fiberglass blanket above and below the panel, independent measurements are required. When such measurements are made, they are typically the average of eight locations over the surface of each panel. A summary of the measured panel thicknesses is shown in Fig. 3. The data show a variation from -6 to $+11 \%$ relative to the nominal value of 1 in. Because vacuum insulation panels are nonhomogenous, various approaches have been developed to determine their overall thermal effectiveness. One method employs an overall hot box technique where mathematical models are used to correct for the effects of materials used to surround the test panel. That method has not yet been tested with the round robin specimens.

The other method in common use employs a finite difference model of the panel, and requires a priori knowledge of the barrier thermal conductivity, the thermal 


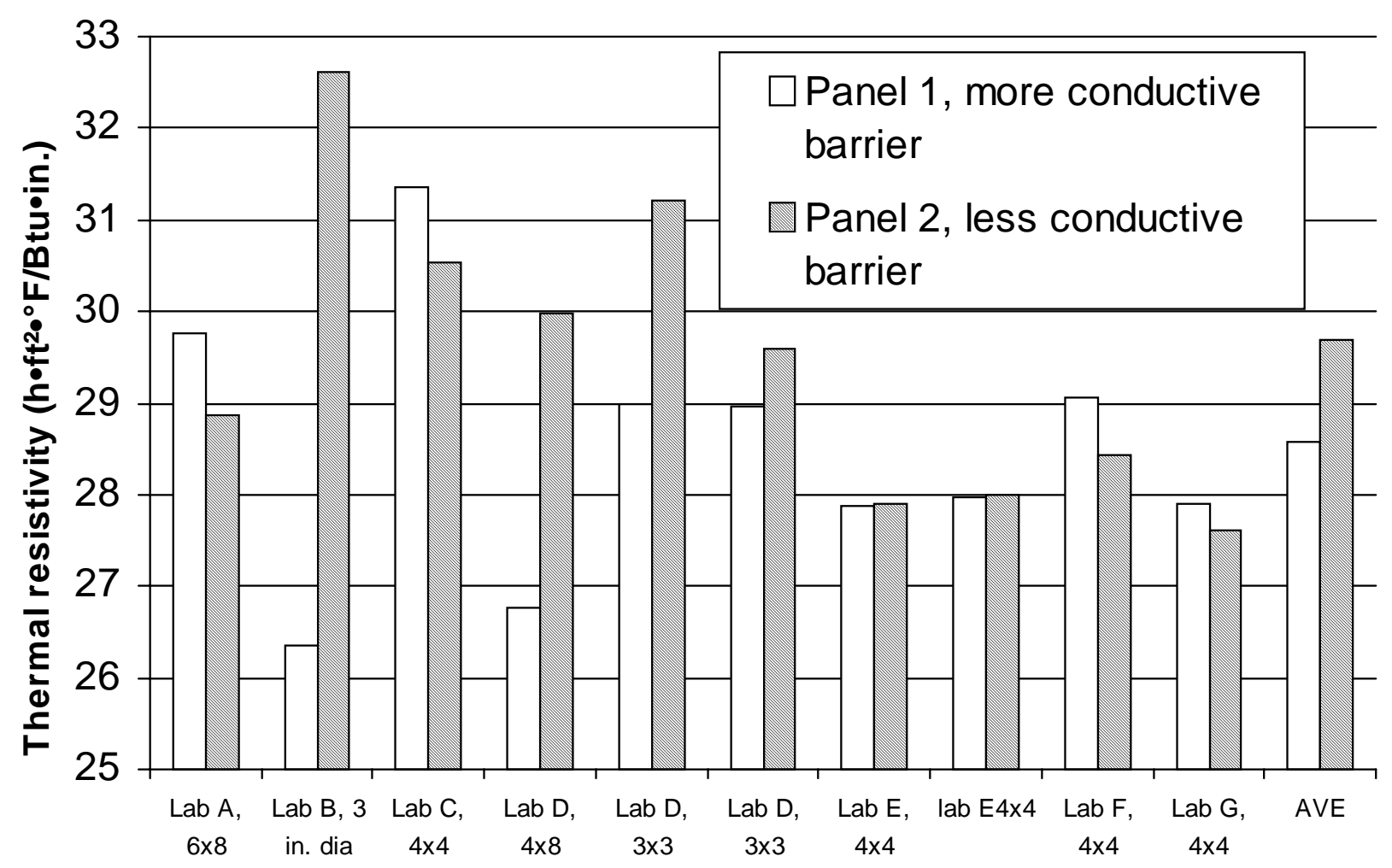

Figure 1. Measured center-of-panel thermal resistivity

conductivity of the evacuated region within the panel, and some definition of the thermal boundary conditions for the analysis. That latter method was used by three of the participating laboratories and the results are shown in Table 2.

Four boundary conditions were considered. The first represents the typical wall or door of a refrigerator. For this configuration, one side of the panel would face a thin sheet

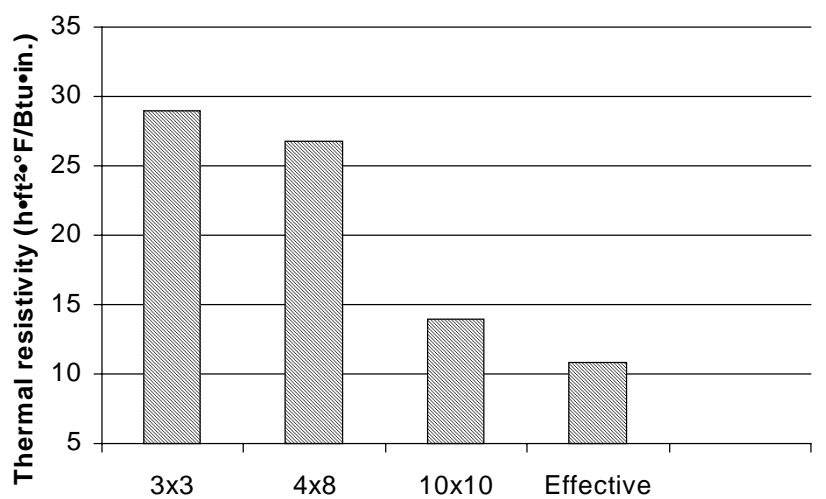

Figure 2. Measured center-of-panel thermal resistivity varies with transducer size for more conductive barrier material, comparison to calculated effective whole panel thermal resistance. of steel $(0.0006 \mathrm{~m}$ thick, $69.23 \mathrm{~W} / \mathrm{m}-\mathrm{K})$ which is in turn exposed to indoor convective transfer to an environment at 21C. The other side of the panel would be surrounded by $2.54 \mathrm{~cm}$. of foam $(0.024 \mathrm{~W} / \mathrm{m}-\mathrm{K})$ and a thin sheet of ABS $(0.003048 \mathrm{~m}$ thick, $0.2596 \mathrm{~W} / \mathrm{m}-\mathrm{K})$ exposed to an air temperature of $4 \mathrm{C}$. The second boundary condition represents a wall section of a building. In that wall, one side of the panel would face $1.27 \mathrm{~cm}$. gypsum board $(0.16$ $\mathrm{W} / \mathrm{m}-\mathrm{K}$ ) exposed to indoor convective conditions (21C) . The other side would face $1.27 \mathrm{~cm}$ of foam $(0.03 \mathrm{~W} / \mathrm{m}-\mathrm{K}))$,

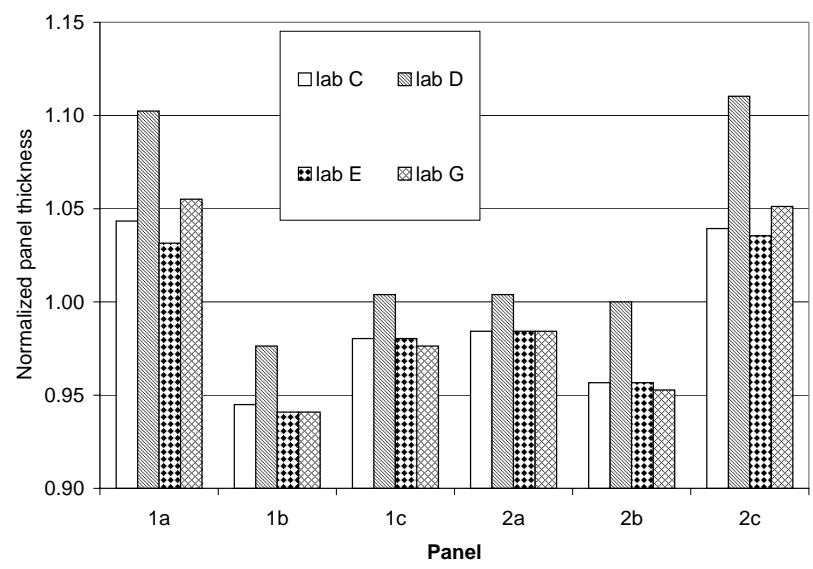

Figure 3. Comparison of measured panel thickness, normalized relative to nominal 1 in. thickness. 


\begin{tabular}{|c|c|c|c|}
\hline \multicolumn{3}{|c|}{ Table 2. Finite Difference/Element Model Results for Whole Panel Thermal Resistance } \\
\hline \multirow{2}{*}{ Model used } & Boundary conditions & \multicolumn{2}{|c|}{ Whole panel thermal resistance $\left(\mathrm{h} \bullet \mathrm{ft}^{2} \bullet{ }^{\circ} \mathrm{F} / \mathrm{Btu}\right)$} \\
\cline { 3 - 4 } & & barrier: more conductive & barrier: less conductive \\
\hline Lab B & Refrigerator door & 10 & 26 \\
\hline Lab D & Refrigerator door & 10 & 23 \\
\hline Lab B & Wall & 12 & 32 \\
\hline Lab D & Wall & 10 & 27 \\
\hline Lab D & Test apparatus & 11 & 27 \\
\hline Lab C & Undefined & 13 & 27 \\
\hline
\end{tabular}

followed by a thin cladding (wood, $2.5 \mathrm{~cm}$. thick, 0.19 $\mathrm{W} / \mathrm{m}-\mathrm{K}$ ) exposed to external convection at $-7 \mathrm{C}$. The third boundary condition is a bit simpler, because it represents a heat flux meter apparatus with standard high-density fiberglass surrounding the panel which is in turn encased within two constant temperature plates. The fourth boundary condition was not known by the laboratory, because they employed a canned program provided to them by others.

Considering the different mathematical models, the different values used for the element conductivities, and the different boundary condition implementations, there is a surprising degree of agreement. The whole panel thermal resistance for the more conductive barrier was 11.2 $\mathrm{h} \bullet \mathrm{ft}^{2} \bullet{ }^{\circ} \mathrm{F} / \mathrm{Btu}$ with a standard deviation of $11 \%$. The whole panel thermal resistance for the less conductive barrier was $26.9 \mathrm{~h} \bullet \mathrm{ft}^{2} \bullet^{\circ} \mathrm{F} / \mathrm{Btu}$ with a standard deviation of $12 \%$.

\section{FUTURE PLANS}

Considering the exploratory nature of this round-robin, and the complexity of the measurements, the results showed surprisingly good agreement. However, there is still much to learn. Future plans to expand the round robin include the addition of more measurements and more laboratories. Specifically, a standard foam board has been added to the round robin to allow us to compare the baseline performance of all the included test apparatus devices. This board will be circulated to all the participating laboratories. There are also several other laboratories that have asked to be included in the program and measurements will be completed using their apparatus and methodologies as soon as possible.

One of the most important future efforts will be the addition of calorimetric measurements at a facility dedicated to such work. This will provide an invaluable benchmark for the finite element modeling efforts.

Another important issue to be addressed is that of the low heat flux calibration. Many of these test devices are typically used to measure insulation with lower resistivity, so the heat flux is usually much greater than that measured during these tests. Special calibration procedures have been developed and reported by some participants, and efforts will be made to determine procedures used by others.

The ultimate goal of the round robin is to determine which test methods give the most useful results, and to provide a definition of the expected accuracy. The information gathered during this effort will lead us to improved industry consensus standard test methods for vacuum insulation panels.

\section{REFERENCES}

1. ASTM C1484-00, Standard Specification for Vacuum Insulation Panels, Annual book of ASTM Standards, Vol.

04.06, West Conshohocken, PA.

\section{BIOGRAPHIES}

\section{Andrzej Brzezinski}

Andrzej Brzezinski, LaserComp's President and Product Development Director, has been in the thermal conductivity business in a variety of capacities for more than 20 years. After graduating from the University of Illinois in 1977 with a degree in Material Science, he worked at Foxborough Corporation before taking a position at Dynatech as Manager in their Testing Laboratory. He then ventured into Sales, Research and Development (he developed a LaserFlash System still being sold) and Management at the highest levels. When Mr. Brzezinski created LaserComp he utilized his experience in running a testing lab and then sales to pin point the needs of the marketplace. Years in design and development provided the technical background to determine the feasibility of wish lists for instruments. All of this resulted in the creation of the FOX Instrument Line, the finest Thermal Conductivity Measurement Instruments in the world 


\section{Therese K. Stovall}

Therese K. Stovall has worked at Oak Ridge National Laboratory since 1978. She is currently the manager of the Engineering Science Section in the Engineering Technology Division. This section performs work in thermal hydraulics, structural mechanics, and irradiation engineering. Previously, Therese worked on distributed generation and insulation programs. She updated the DOE Insulation Fact Sheet, the second most requested document published by DOE, and has contributed to laboratory evaluations of a variety of insulation materials, focusing her efforts on evacuated insulation panels. Previously, Therese managed the Ice Storage Test Facility, a commercial ice storage testing program sponsored by the Electric Power Research Institute. She received a patent for a load management method using wallboard containing a phase change material.

Therese Stovall received her B.S.M.E. with distinction from Purdue University in 1977, her M.S.M.E. from the University of Tennessee in 1988 and is a Licensed Professional Engineer in the state of Tennessee. She is also a member of the American Society of Mechanical Engineers, the American Society for Testing and Materials, and the American Society of Heating Refrigerating and Air Conditioning Engineers. 